\title{
ASTHMA
}

\section{Intravenous salbutamol bolus compared with an aminophylline infusion in children with severe asthma: a randomised controlled trial}

\author{
G Roberts, D Newsom, K Gomez, A Raffles, S Saglani, J Begent, P Lachman, K Sloper, \\ R Buchdahl, A Habel on behalf of the North West Thames Asthma Study Group
}

Thorax 2003;58:306-310

See end of article for authors' affiliations

Correspondence to: Dr G Roberts, Paediatric Respiratory Medicine, Royal London Hospital, London El 1BB; g.c.roberts@ic.ac.uk

Revised version received 2 December 2002 Accepted for publication 16 December 2002

\begin{abstract}
Background: The relative efficacies of aminophylline and salbutamol in severe acute childhood asthma are currently unclear. A single bolus of salbutamol was compared with a continuous aminophylline infusion in children with severe asthma in a randomised double blind study.

Methods: Children aged 1-16 years with acute severe asthma were enrolled if they showed little improvement with three nebulisers (combined salbutamol and ipratropium) administered over an hour and systemic steroids. Subjects were randomised to receive either a short intravenous bolus of salbuta$\mathrm{mol}(15 \mathrm{\mu g} / \mathrm{kg}$ over 20 minutes) followed by a saline infusion or an aminophylline infusion $(5 \mathrm{mg} / \mathrm{kg}$ over 20 minutes) followed by $0.9 \mathrm{mg} / \mathrm{kg} / \mathrm{h}$.

Results: Forty four subjects were enrolled, with 18 randomly allocated to receive salbutamol and 26 to receive aminophylline. The groups were well matched at baseline. An intention to treat analysis showed that there was no statistically significant difference in the asthma severity score (ASS) at 2 hours between the two groups (median (IQR) $6(6,8)$ and $6.5(5,8)$ for salbutamol and aminophylline respectively, $p=0.93$ ). A similar improvement in ASS to 2 hours was seen in the two groups (mean difference $-0.08,95 \% \mathrm{Cl}-0.97$ to 0.80 ), there was a trend $(p=0.07)$ towards a longer duration of oxygen therapy in the salbutamol group $(17.8$ hours $(95 \% \mathrm{Cl} 8.5$ to 37.5$) \vee 7.0$ hours $(95 \% \mathrm{Cl} 3.4$ to $14.2)$, and a significantly ( $p=0.02)$ longer length of hospital stay in the salbutamol group $185.4195 \%$ $\mathrm{Cl} 66.1$ to 110.2 ) hours $v 57.3$ hours $(95 \% \mathrm{Cl} 45.6$ to 72.0$)$ ). There was no significant difference in adverse events between the two groups.

Conclusions: This study suggests that, in severe childhood asthma, there is no significant difference in the effectiveness of a bolus of salbutamol and an aminophylline infusion in the first 2 hours of treatment. Overall, the aminophylline infusion was superior as it significantly reduced the length of stay in hospital.
\end{abstract}

$\mathrm{T}$ he consensus treatment for severe acute exacerbations of asthma is a combination of nebulised $\beta$ agonist and ipratropium with oral corticosteroids. ${ }^{12}$ There is controversy as to whether aminophylline or a $\beta_{2}$ agonist such as salbutamol is the most effective first line intravenous bronchodilator for the optimal management of a child who does not improve. ${ }^{3}$ Many British paediatricians consider that aminophylline is the drug of choice, ${ }^{1}$ despite the limited evidence for its efficacy and significant adverse effects. ${ }^{4-9}$ Intravenous salbutamol has a better safety profile and has been shown to reduce the severity and duration of severe acute asthma in children..$^{10}$ More recently it has also been shown in two studies that salbutamol is effective in acute severe asthma when given as a short bolus in combination with continuous nebulised salbutamol. ${ }^{11}{ }^{12}$ One hypothesis for this benefit is that intravenous salbutamol reaches airways obstructed by the bronchospasm, oedema and mucus associated with a severe exacerbation of asthma; once opened, nebulised agents may then reach these airways. Intravenous salbutamol and aminophylline have not been compared in a study of sufficient size to detect differences in their effectiveness. ${ }^{13}$ In this study we compare the effectiveness of a short bolus of salbutamol with an aminophylline infusion in children and teenagers with severe asthma using a randomised double blind design.

\section{METHODS}

\section{Study population}

Subjects were recruited between 1999 and 2001 from five district general hospitals in the North West Thames region (West
Middlesex University Hospital, Queen Elizabeth II Hospital, Northwick Park Hospital, Ealing Hospital, and Hillingdon Hospital). Subjects were included if they were aged 1-16 years and had presented with acute severe asthma that had responded poorly to three nebulisers containing salbutamol ( $2.5 \mathrm{mg}, 5 \mathrm{mg}$ if $\geqslant 5$ years) and ipratropium ( $125 \mu \mathrm{g}, 250 \mu \mathrm{g}$ if $\geqslant 5$ years) over a 1 hour period. Asthma was diagnosed on the basis of clinical history and examination. ${ }^{14}$ A score of 7 or more on the 9-point asthma severity score (ASS) indicated a severe exacerbation. ${ }^{15}{ }^{16}$ A poor response was defined as an improvement in the ASS of $<2$; an ASS after three doses of nebulised treatment of $\geqslant 7$; or a continuing requirement for supplementary oxygen to maintain saturations of at least $92 \%$ (Ohmeda 3800 pulse oximeter). Subjects were excluded if they had a life threatening exacerbation, an underlying respiratory disease other than asthma, cardiac disease, or treatment with a medication that alters the metabolism of aminophylline.

Written consent was obtained from all families and the study was approved by the Thames Multicentre Research ethics committee and the local ethics committees.

\section{Intervention}

Subjects were treated with either a single bolus of intravenous salbutamol ( $15 \mu \mathrm{g} / \mathrm{kg}$ over 20 minutes) followed by an infusion of saline or a continuous aminophylline infusion (bolus of $5 \mathrm{mg} / \mathrm{kg}$ over 20 minutes followed by an infusion of $0.9 \mathrm{mg} / \mathrm{kg} / \mathrm{h}$ ). Visibly identical numbered treatment packs were made by the pharmacy department at West Middlesex 


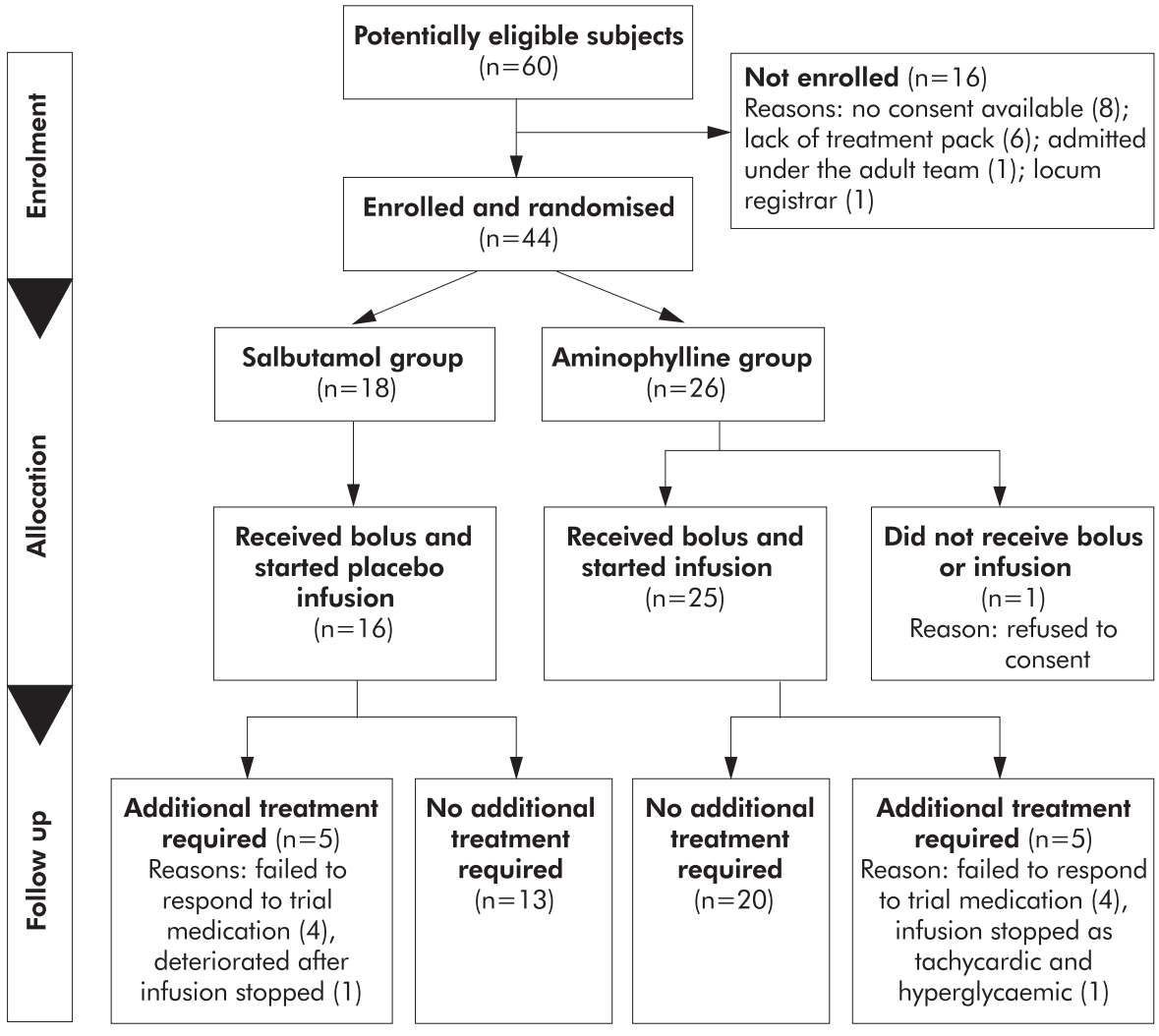

Figure 1 Subject flow sheet.

University Hospital. Study numbers were assigned to aminophylline or salbutamol according to a random number table. Each centre had its own sequence of numbers to ensure that each enrolled similar numbers into each group. Subjects were treated with the next serially numbered treatment pack. Only one investigator (GR), who had no involvement in the enrolment or clinical care of any subject, was aware of this allocation. Blood was taken 1 hour after starting intravenous therapy for measurement of potassium and aminophylline levels. The drug levels were assayed centrally and checked by one investigator (GR). If the drug level was outside the range $7-15 \mathrm{mg} / \mathrm{l}^{17}$ the clinicians were instructed to adjust the infusion rate. For subjects in the salbutamol arm, the clinicians were given instructions to maintain, increase, or reduce the rate at random to maintain blinding. All subjects were also treated with systemic corticosteroids, nebulised salbutamol as required, and 6 hourly nebulised ipratropium. Nebulised and intravenous treatment was reduced according to a regime based on the ASS as follows: score $\geqslant 7$, continuous nebulisation; 6, nebulisation half hourly; 5, nebulisation hourly; 4 , nebulisation every 2 hours; score of $\leqslant 3$ and out of oxygen, 4 hourly treatment and infusion stopped. Where the treating clinicians considered that a subject was not improving, the treatment allocation was unblinded and additional treatment commenced. If subjects had tolerated 4 hourly nebulisers overnight, the following morning the paediatricians were instructed to switch to inhaled treatment and to discharge them that afternoon if inhalers were well tolerated.

\section{Outcome measures}

The ASS was assessed just before the start of intravenous treatment and at 1, 2, 6, 12, and 24 hours. Ventilated subjects were given an ASS of 9. The 9-point ASS was validated within the study; the mean difference (range) in the score assigned by each of two observers was $0.1(-1$ to +1$) .{ }^{18}$ Saturation in air was also recorded at hourly intervals before nebulised treatment to determine when supplementary oxygen was no longer required to keep the saturations at $92 \%$ or above (Ohmeda 3800). Subjects were taken off oxygen for 5 minutes before the measurement. Where the saturation dropped below $85 \%$, the oxygen was restarted and the reading recorded as less than $85 \%$. Peak expiratory flow measurements were not routinely recorded as most of the subjects were unable to perform this because of their age or the severity of the exacerbation. Adverse effects were recorded. All outcome measures were recorded by staff who were unaware of the subject's allocation.

\section{Statistical methods}

An intention to treat analysis was undertaken. The ASS in each group after 2 hours of treatment was compared using a Wilcoxon rank sum test (early primary end point). The change in the ASS from baseline to 2 hours was normally distributed; this parameter was compared between the two groups to provide a comparison that included a confidence interval. The duration of supplementary oxygen therapy (late primary end point) and the time to discharge (secondary end point) in each group were compared using an unpaired $t$ test. In addition, the percentage of subjects experiencing an adverse event in each group was compared using a $\chi^{2}$ or Fisher's exact test, as appropriate (secondary end point) and the serum potassium level before and after the bolus was compared using a $t$ test. It was calculated that the data from 42 subjects would be sufficient to detect a $30 \%$ difference in change in severity score at 2 hours between the two groups assuming a standard deviation of $30 \%$ of the ASS, $90 \%$ power, and a $5 \%$ level of significance. An interim analysis was undertaken by one investigator (GR) after 21 subjects had been randomised, with a plan to stop the study if the primary end point reached statistical significance at a level of $<0.001$ (Peto method). This criterion was not reached. The other investigators were blind to this interim analysis. A level of $5 \%$ was taken as significant 
Table 1 Baseline characteristics of study subjects

\begin{tabular}{|c|c|c|c|}
\hline Item & Salbutamol $(n=18)$ & Aminophylline $(n=26)$ & p value* \\
\hline$M: F(\%$ males $)$ & $12: 6(66.7 \%)$ & $20: 6(76.9 \%)$ & 0.45 \\
\hline Age (years) & $3.85(1.35,15.55)$ & $4.12(1.19,13.13)$ & 0.80 \\
\hline Weight $(\mathrm{kg})$ & $15.0(12.0,30.4)$ & $16.8(12.5,30.0)$ & 0.83 \\
\hline Age at which asthma diagnosed (years) & $2.00(1.00,2.75)$ & $1.25(1.00,2.75)$ & 0.63 \\
\hline No of previous admissions with asthma & $1(0,2)$ & $1(0,4)$ & 0.54 \\
\hline Previous intravenous therapy for asthma & $4(22.2 \%)$ & $4(15.4 \%)$ & 0.70 \\
\hline Previous ventilatory support for asthma & $1(5.6 \%)$ & $1(3.9 \%)$ & 1.00 \\
\hline No with eczema & $8(88.8 \%)$ & $8(72.7 \%)$ & 1.00 \\
\hline Duration of exacerbation (hours) & $24(24,72)$ & $24(24,48)$ & 0.36 \\
\hline Treatment with nebulised $\beta$ agonists before presentation & $2(11.8 \%)$ & $5(20.0 \%)$ & 0.68 \\
\hline Duration of treatment with systemic corticosteroids pre-study bolus (hours) & $3.0(0.5,10.9)$ & $0.0(0.0,3.5)$ & 0.06 \\
\hline ASS on admission & $8(6,9)$ & $9(7,9)$ & 0.45 \\
\hline ASS at start of study bolus & $8(7,8)$ & $8(7,9)$ & 0.76 \\
\hline Saturation in air on admission & $87.5 \%(<84.0,91.0)$ & $90.0 \%(87.0,94.0)$ & 0.26 \\
\hline Saturation in air at start of study bolus & $91.0 \%(88.5,93.5)$ & $91.0 \%(88.0,93.0)$ & 0.77 \\
\hline Need for supplementary oxygen at start of trial bolus & $14(77.8 \%)$ & $19(79.2 \%)$ & 0.91 \\
\hline
\end{tabular}

Values are medians with interquartile ranges or proportions with percentages.

*Comparison of subjects in each group: medians compared with Wilcoxon rank sum test; proportions compared with $\chi^{2}$ or Fisher's exact test as appropriate.

Table 2 Progress of study subjects

\begin{tabular}{|c|c|c|c|c|}
\hline Item & Salbutamol $(n=18)$ & Aminophylline $(n=26)$ & Difference $(95 \% \mathrm{Cl})$ & $p$ value* \\
\hline Asthma severity score (ASS) at 2 hours & $6(0.37)$ & $6.5(0.33)$ & $0.5(-)$ & 0.93 \\
\hline Change in ASS from time 0 to 2 hours & $-1.11(0.39)$ & $-1.19(0.25)$ & $-0.08(-0.97$ to 0.80$)$ & 0.85 \\
\hline Duration of oxygen therapy (hours) & $17.8(8.9)$ & $7.0(6.1)$ & 2.56 times longer $(0.92$ to 7.18$)$ & 0.07 \\
\hline Duration of infusion (hours) & $32.2(3.2)$ & $27.8(0.85)$ & $-4.4(-6.2$ to 14.8$)$ & 0.41 \\
\hline Time to discharge (hours) & $85.4(13.2)$ & $57.3(8.5)$ & 1.49 times longer $(1.06$ to 2.10$)$ & 0.02 \\
\hline
\end{tabular}

Values for the sulbutamol and aminophylline groups are presented with standard errors (SE).

* Comparison of subjects in each group using unpaired $t$ test (change in ASS, duration of oxygen and infusion, and time to discharge) or Wilcoxon rank sum test (ASS at 2 hours).

All subjects included in the intention to treat analysis.

for the final analysis. All statistical tests were undertaken using Stata 6.

\section{RESULTS}

\section{Subjects and treatment allocation}

Sixty children were admitted with severe asthma during a cumulative recruitment period of 97 months at the five hospitals, 44 of whom were enrolled into the study (fig 1). Eighteen subjects $(40.9 \%)$ were randomly allocated to treatment with a bolus of salbutamol and $26(59.1 \%)$ to an aminophylline infusion. There were no significant differences in the baseline demographic characteristics of these subjects, in their previous asthma history or presenting exacerbation (table 1). There were three early withdrawals; one from the aminophylline group refused to allow a cannula to be inserted and two in the salbutamol group were given additional treatment before the initial intravenous bolus finished because of a rapid clinical deterioration.

Mean serum aminophylline levels taken 1 hour after commencing the bolus dose were $9.1 \mathrm{mg} / \mathrm{l}$ (95\% CI 8.4 to 12.3). One subject with a level below 7 (6.9 mg/l) failed to respond to the infusion and received an infusion of salbutamol after 7.5 hours. Two subjects had levels above $15 \mathrm{mg} / \mathrm{l}(21.2$ and 25.5); one experienced vomiting. Both their infusions were temporarily stopped and restarted at a lower rate.

\section{Asthma severity score}

Using an intention to treat analysis, there were no significant differences in the ASS for each group either before the commencement of the intravenous bronchodilator or in the following 24 hours (table 2, fig 2). The difference in the change in ASS between the aminophylline and salbutamol groups was -0.08 ( $95 \%$ CI -0.97 to 0.80$)$, table 2 . This result was unchanged when the early withdrawers were excluded.

\section{Supplementary oxygen}

There was no difference in the proportions requiring supplementary oxygen or the saturation in air before intravenous treatment (table 2) or at any time in the subsequent 24 hours (fig 3). Subjects in the aminophylline and salbutamol groups required supplementary oxygen for 7.0 hours (95\% CI 3.4 to 14.2 ) and 17.8 hours (8.5 to 37.5 ), respectively (table 2 ). The salbutamol group therefore required supplementary oxygen for 2.56 times longer (95\% CI 0.92 to 7.18) using an intention to treat analysis. Exclusion of the early withdrawals from the analysis did not change the result.

\section{Additional therapy}

The intravenous study medication ran for similar lengths of time in the salbutamol and aminophylline groups (table 2). The use of nebulised salbutamol was also similar in the two groups with at least hourly treatment for the first 6 hours

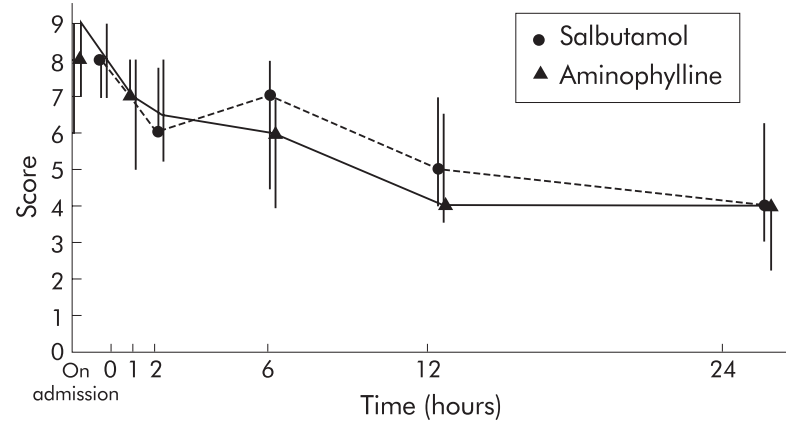

Figure 2 Asthma severity scores in the salbutamol (dotted line) and aminophylline (continuous line) groups. Values are plotted as medians with interquartile ranges. 


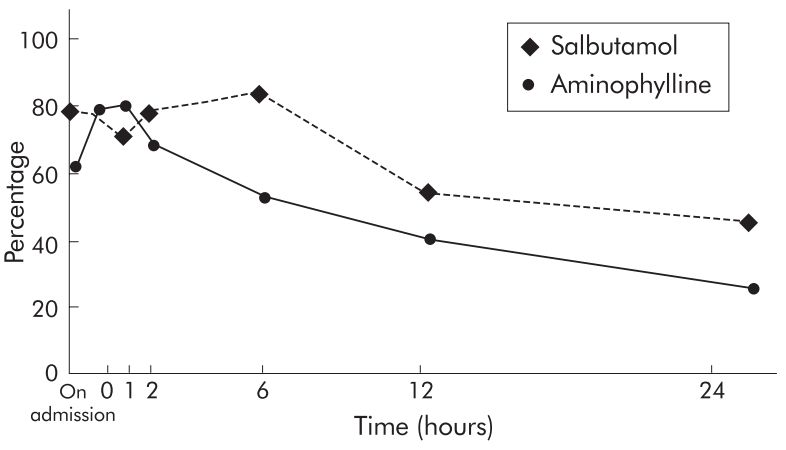

Figure 3 Percentage requiring supplementary oxygen to maintain saturations at $92 \%$ or above in the salbutamol (dotted line) and aminophylline (continuous line) groups.

(data not shown). Five (27.8\%) subjects from the salbutamol group and four (15.4\%) subjects from the aminophylline group required additional infusions of aminophylline, salbutamol, or magnesium sulphate. Two subjects (11\%) in the salbutamol group and one (4\%) in the aminophylline group also required ventilation. All responded to further treatment. Thirteen $(72.2 \%)$ subjects in the salbutamol group and $20(84.6 \%)$ in the aminophylline group required no additional treatment. There were no significant differences in the demographic details, asthma history, or presenting features before the commencement of the intravenous bolus between subjects requiring additional treatment and the other subjects (data not shown).

\section{Discharge}

There was a significant difference $(p=0.02)$ in the time from the commencement of intravenous treatment to discharge from hospital in the two groups (table 2), with the duration of inpatient treatment for the salbutamol group being 1.49 times longer (95\% CI 1.06 to 2.10 ) than the aminophylline group.

\section{Adverse events}

The most frequent adverse events were nausea, vomiting, and abdominal pain. There were no significant differences in the number of adverse events reported by the salbutamol and aminophylline groups $(22.2 \% \vee 36 \%, p=0.50$, Fisher's exact test). There was no significant change in the mean serum potassium level with either intravenous treatment; $5-10 \%$ of subjects had a serum potassium level $<3 \mathrm{mmol}$ before starting either intravenous bronchodilator and a similar proportion were hypokalaemic in the few hours after starting intravenous treatment (data not shown).

\section{DISCUSSION}

In this study we have compared an intravenous bolus of salbutamol with an infusion of aminophylline in a population of children with severe acute asthma that was unresponsive to maximal nebulised treatment and systemic corticosteroids. The results show that there was no statistically significant difference in the effectiveness of these two regimes after 2 hours, although overall the aminophylline infusion appeared superior as it reduced the length of stay in hospital.

A recent Cochrane review compared aminophylline with placebo in children with severe acute asthma receiving treatment with inhaled bronchodilators and systemic glucocorticoids. ${ }^{19}$ It showed that aminophylline improves lung function, reduces clinical severity, and reduces the need for ventilation. This is contrary to the results of a previous metaanalysis of adult studies. ${ }^{20}$ The largest study included in the Cochrane review was by Yung et al ${ }^{8}$ which contributed 163 of the 380 cases and was the only one to show a measurable benefit. Like the study by Yung et al, our investigation enrolled subjects only if they had a severe exacerbation of asthma. This suggests that subjects with less severe exacerbations improve regardless of whether they receive additional intravenous treatment, and that the relatively small improvement in lung function produced by an aminophylline infusion is therefore only beneficial to children with the most severe exacerbations. The study presented here also showed that the group receiving aminophylline had a 30\% shorter stay in hospital. This has significant implications for health service resources as well as the child's well being, given that an aminophylline infusion appears to shorten the admission time by more than a day.

A second Cochrane review ${ }^{13}$ which examined the addition of intravenous salbutamol in severe acute asthma in adults and children found that it afforded no additional benefit over inhaled treatment or placebo. These conclusions are flawed for a number of reasons; $80 \%$ of the included studies were published before 1990 when lower doses of intravenous $\beta_{2}$ agonists were used compared with those currently in use. ${ }^{21}$ The review states that there are insufficient paediatric studies to provide subgroup comparisons. This is important as children with asthma behave differently from adults as atopy is a more common feature and they do not have concurrent chronic obstructive airway disease or ischaemic heart disease. All but one of the studies in the Cochrane review used suboptimal concurrent nebulised therapy. The two adequately sized randomised controlled trials in children show that intravenous salbutamol is effective. ${ }^{1011}$ Brown et al ${ }^{11}$ enrolled subjects who failed to improve after one dose of nebulised salbutamol. A bolus of intravenous salbutamol or placebo was followed by frequent nebulised therapy. A significant improvement in the ASS occurred within 2 hours, together with earlier discharge from hospital. Intravenous salbutamol may be able to reach the obstructed airways seen in severe exacerbations of asthma, thereby allowing nebulised agents to reach them.

Intravenous aminophylline and $\beta_{2}$ agonist have only been compared in severe acute childhood asthma in one study. ${ }^{22}$ This study used very small intravenous dosages, gave no concurrent nebulised therapy, and enrolled insufficient subjects to detect anything but a major difference. The adult studies addressing this issue are similarly flawed. ${ }^{13}$ Using the currently recommended intravenous dosages and frequency of concurrent nebulised therapy, ${ }^{12}$ we have shown that an aminophylline infusion is more effective than a single bolus of salbutamol. Only three quarters of the salbutamol group recovered with a single bolus, which is disappointing as a bolus of salbutamol ${ }^{11}$ is an attractive option for the busy paediatric emergency department. The subjects enrolled into the study by Browne et $a l^{11}$ had only been treated with one dose of nebulised salbutamol whereas our patients had failed to improve with three doses of nebulised salbutamol and ipratropium. It is therefore perhaps not surprising that, unlike Browne et al, we failed to show that a single bolus of salbutamol was more effective than aminophylline. It is possible, however, that a proportion of these non-responders would have improved after a further bolus or a continuous infusion of salbutamol.

This study has several limitations. It is relatively small, although we were still able to demonstrate equivalence after 2 hours and a significant difference in duration of admission. The $95 \%$ confidence interval for the difference in improvement in ASS to 2 hours between the two groups was -0.97 to 0.80 . Given that the ASS is a 9 point scoring system, the difference in efficacy between the two treatments is minimal within this time frame. A larger study would have enabled a comparison of other important outcome measures such as the need for mechanical ventilation, although with only $11 \%$ and $4 \%$ requiring ventilation in the salbutamol and aminophylline groups, respectively, a few hundred subjects would have had to be enrolled to address this issue successfully. Although there was an imbalance in the allocation between the two groups, this did not adversely reduce the statistical power of the study. 
We included subjects who satisfied the clinical severity score, regardless of whether or not they required supplementary oxygen. This reduced the number of subjects available to determine the relative length of supplementary oxygen therapy, which probably prevented this end point reaching significance. Aminophylline levels were only assayed after 1 hour of treatment when many of the subjects had levels below the quoted therapeutic range. They had, however, received the standard aminophylline loading dose. ${ }^{1}$ It is perhaps significant that the two studies which showed that aminophylline is effective used a higher bolus dose. ${ }^{8}{ }^{9}$ However, we have shown that an infusion of aminophylline is more effective than a bolus of salbutamol. As we did not provide a protocol for treating subjects who failed to improve with the study medication, we were unable to examine systematically the role of adding the other bronchodilator or additional agents such as magnesium sulphate.

The consensus for treating severe acute asthma is currently moving from intravenous aminophylline towards intravenous $\beta_{2}$ agonist. ${ }^{23}$ This is despite the paucity of randomised controlled trials incorporating current dosages of intravenous bronchodilators and frequent nebulised therapy in the paediatric age group. This study is helpful in informing the debate on the relative merits of intravenous salbutamol and aminophylline and whether salbutamol should be used as a bolus or infusion. It shows that there is no statistically significant difference in the effectiveness of intravenous salbutamol and aminophylline over the first 2 hours of treatment. Overall, the infusion of aminophylline was more effective with a significant reduction in the duration of admission and a trend towards a shorter duration of supplementary oxygen. Adverse effects did not appear to be significantly greater in the aminophylline group. Further clinical studies are required to determine whether multiple intravenous boluses of salbutamol are as effective as an aminophylline infusion, to evaluate the relative effectiveness of infusions of salbutamol and aminophylline, and to investigate whether these two intravenous bronchodilators are synergistic in acute severe asthma.

\section{ACKNOWLEDGEMENTS}

The authors acknowledge the help of all the staff working at the five hospitals involved, particularly Andrea Thomas, Pippa Banister; Mitch Blair, Wouter Hanekom, Keith Brent, Nitha Naqvi, Julian Redhead, Kecia Taylor and Ranjan Suri; the statistical advice provided by Paul Bassett; and all the children and families involved without whom the study would not have been possible.

Authors' affiliations

G Roberts, Imperial College School of Medicine at St Mary's Hospital, London, UK

G Roberts, K Gomez, A Habel, West Middlesex Hospital

D Newsom, P Lachman, Northwick Park Hospital

A Raffles, S Saglani, J Begent, Queen Elizabeth II Hospital

K Sloper, Ealing Hospital

R Buchdahl, Hillingdon Hospital
DN had the original idea for the study. The protocol was developed by $G R, D N$ and $A H$. All the authors were involved in entering subjects into the study and collecting data. GR undertook the data analysis and wrote the first draft of the paper. All the authors contributed to the revision of the paper. AH will act as guarantor of the paper.

This work was supported by funding from the NHS London Regional Office, Research \& Development Programme. The views expressed in the publication are those of the authors and not necessarily of the NHS or the Department of Health.

\section{REFERENCES}

1 British Thoracic Society, National Asthma Campaign, Royal College of Physicians of London, et al. The British guidelines on asthma management: 1995 review and position statement. Thorax 1997;52(Suppl 1):S1-21.

2 Qureshi F, Pestian J, Davis P, et al. Effect of nebulised ipratropium on the hospitalisation rates of children with asthma. N Engl J Med 1998;339:1030-5.

3 McKenzie S. Aminophylline in the hospital treatment of children with acute asthma. BM 1994;308:1384-5.

4 Strauss R, Wetheim D, Bonagura V. Aminophyline therapy does not improve outcome and increases adverse effects in children hospitalised with acute asthmatic exacerbations. Pediatrics 1994;93:205-10.

5 Zainudin B. Effect of adding aminophylline to nebulised salbutamol in severe acute asthma. Thorax 1994;49:267-9.

6 Rodrigo C. Rodrigo G. Treatment of acute asthma. Lack of therapeutic benefit and increase of the toxicity from aminophylline given in addition to high doses of salbutamol delivered by metered dose inhaler via a spacer. Chest 1994,106:1071-6.

7 Littenberg B. Aminophylline treatment in severe acute asthma. A meta analysis. JAMA 1988;259:1678-84.

8 Yung $M$, South M. Randomised controlled trial of aminophylline for severe acute asthma. Arch Dis Child 1998;79:405-10.

9 Ream RS, Loftis LL, Albers GM, et al. Efficacy of iv theophylline in children with severe status asthmaticus. Chest 2001;119:1480-8.

10 Kirby C. Comparison of intravenous and inhaled salbutamol in severe acute asthma. Pediatr Rev Commun 1988;3:67-77.

11 Browne GJ, Penna AS, Phung X, et al. Randomised trial of intravenous salbutamol in early management of acute asthma in children. Lancet 1997;349:301-5.

12 Browne GJ, Trieu L, Van Asperen P. Randomized, double-blind, placebo-controlled trial of intravenous salbutammol and nebulized ipratropium bromide in early management of severe acute asthma in children presenting to an emergency department. Crit Care Med 2002;30:448-53

13 Travers A, Jones AP, Kelly K, et al. Intravenous beta2-agonists for acute asthma in the emergency department (Cochrane Review). In: Cochrane Library. Issue 4. Oxford: Update Software, 2001.

14 American Thoracic Society. Standards for the diagnosis and care of patients with chronic obstructive pulmonary disease (COPD) and asthma. Am Rev Respir Dis 1987; 136:225-44.

15 Bishop J, Carlin J, Nolan T. Evaluation of the properties and reliability of a clinical severity scale for acute asthma in children. J Clin Epidemiol 1992;45:71-6

16 Yung $M$, South $M$, Byrt. Evaluation of an asthma severity score. J Paediatr Child Health 1996;32:261-4.

17 Winter ME. Basic clinical pharmacokinetics. 3rd ed. Philadelphia: Lippincott Williams \& Wilkins, 1994.

18 Bland JM. Altman DG. Statistical methods for assessing agreement between two methods of clinical measurement. Lancet 1986;i:307-10.

19 Mitra A, Bassler D, Ducharme FM. Intravenous aminophylline for severe asthma in children over 2 years using inhaled bronchodilators (Cochrane Review). In: Cochrane Library. Issue 4. Oxford: Update Software, 2001

20 Belda J, Parameswaran K, Rowe BH. Additional of intravenous aminophylline to beta2-agonistis in adults with acute asthma (Cochrane Review). In: Cochrane Library. Issue 2. Oxford: Update Software, 2001.

21 Advanced Life Support Group. Advanced paediatric life support: the practical approach. 3rd ed. London: BM Books, 2001

22 Hambleton G. Comparison of IV salbutamol with IV aminophylline in the treatment of severe, acute asthma in childhood. Arch Dis Child 1979;54:391-402.

23 British Thoracic Society/Scottish Intercollegiate Guidelines Network. British guidelines on the management of asthma. Thorax 2003;58(Suppl I):i 1-94. 